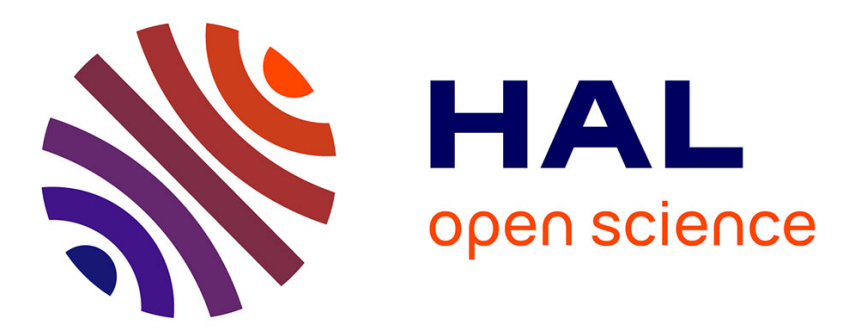

\title{
Development of dairy breeding programmes
}

Didier Boichard

\section{To cite this version:}

Didier Boichard. Development of dairy breeding programmes. Advances in breeding of dairy cattle, 72 , Burleigh Dodds Science Publishing, 2019, Burleigh Dodds Series in Agricultural Science, 978178676 2962 PDF: 978-1-78676-299-3 ePub: 978-1-78676-298-6. 10.19103/AS.2019.0058.28 . hal-02788520

\section{HAL Id: hal-02788520 \\ https://hal.inrae.fr/hal-02788520}

Submitted on 5 Jun 2020

HAL is a multi-disciplinary open access archive for the deposit and dissemination of scientific research documents, whether they are published or not. The documents may come from teaching and research institutions in France or abroad, or from public or private research centers.

Copyright

L'archive ouverte pluridisciplinaire HAL, est destinée au dépôt et à la diffusion de documents scientifiques de niveau recherche, publiés ou non, émanant des établissements d'enseignement et de recherche français ou étrangers, des laboratoires publics ou privés. 


\section{Advances in breeding of dairy cattle}

Edited by Professor Julius van der Werf

University of New England, Australia

Professor Jennie Pryce

Agriculture Victoria and La Trobe University, Australia

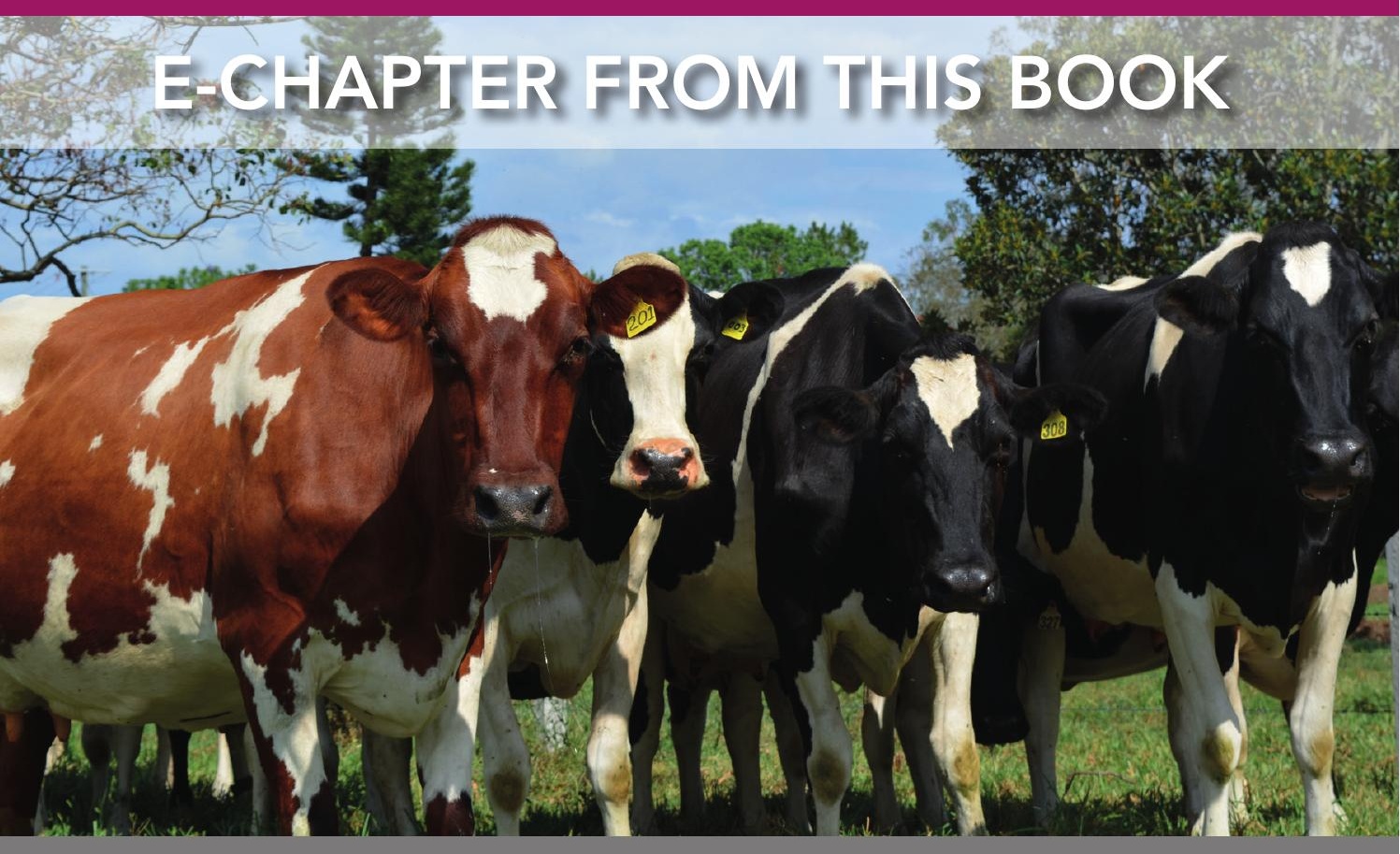

\section{burleigh dodds}




\section{Development of dairy breeding programmes}

Didier Boichard, INRA, AgroParisTech and Université Paris-Saclay, France

1 Introduction

2 The genomic revolution

3 A major change in organization

4 New breeding objectives and genetic trends

5 Genomic selection and genetic diversity

6 Female genotyping

7 Selection for new traits

8 Conclusion and future trends

9 Acknowledgements

10 References

\section{Introduction}

For more than 60 years, a unique strategy has been used worldwide to select dairy cattle. This was the direct consequence of biological characteristics of cows which are unfavourable, such as the low reproductive capacity of females (about 0.4 female progeny per dam per year) and a long generation interval. In addition, in many countries, herds are of small to medium size and always too small to allow efficient selection internally. Reproductive capacity of males is higher, but most traits of interest in dairy cattle are expressed only by females (production, female fertility, udder health, udder conformation, calving ability etc.). Consequently, males cannot be evaluated alone. Hence, without dedicated designs, males only receive pedigree indices with a rather low reliability. In the middle of the twentieth century, the development of artificial insemination provided a tool of choice to design a complex, costly, but very efficient breeding strategy. Indeed, the biological capacity of a bull is outstanding in terms of semen production, and much higher than for all other farm animals. A bull can easily produce tens of thousands of semen straws in a few months. This semen can be frozen for a very long time with limited loss of fertility. Therefore, it is possible to separate semen production and its use 
for reproduction in distant herds several months or years later. This property was used to design the so-called progeny-test schemes which became the reference system worldwide. For each young bull to be evaluated, an initial batch of daughters was produced in a number of commercial herds enrolled in performance recording. Three years later, these daughters were assessed for a number of traits and provided a progeny-based evaluation of the bull. The size of the progeny group varied according to the country, the breed, and the breeding objective. In many breeding schemes, a typical progeny group size was between 80 and 150 daughters, providing a high reliability of the bull's estimated breeding values for moderate-to-high heritable traits. When emphasis was put on low heritable traits (such as fertility or resistance to disease) such as in the Nordic countries, the size of the progeny group was larger. For regional breeds of limited size, the size of the progeny group was smaller (30-50) in order to keep a sufficient number of evaluated young bulls. Based on this progeny evaluation, the best bulls (typically the best $10 \%$ for large breeds) were selected and used on a large scale in the domestic market, and possibly also worldwide. In many breeding schemes, about $10 \%$ of the inseminations were made with young bulls, whereas $90 \%$ were with selected 'service' bulls. Again, in small breeds or when progeny groups of young bulls are larger, this proportion of young bull inseminations could be higher.

This strategy takes a long time. A bull is 5 years old when he gets his first progeny-based evaluations, and 8 years old when the large second batch of daughters start to reproduce. It is also very expensive. In addition to their initial price, the bulls have to be kept up to 5 years of age in perfect sanitary conditions. They have to produce an initial stock of semen and the breeder has to cover the cost of the progeny test, that is the cost of getting daughters phenotypes. In practice, the overall cost was evaluated to be around 40-50 k€/ bull. As only one out of ten was selected for wide use, the cost of each selected bull reached around $500 \mathrm{k} €$, a value which can be paid back only by the sale of tens of thousands of semen doses.

In order to maximize the probability of getting an elite bull after the progeny test, the initial selection steps - which are less costly - were very intense. Indeed, each young bull was born from the best bulls and the best dams available in the market.

Such a strategy strongly relied on a high-quality large-scale information system. Progeny must be identified and both their parents known; all of their performances, for a variety of traits, must be recorded as well as those of their herdmates. Selection being a long process, this information must be stored long term in a homogeneous way. Because all these performances were obtained in different herds and variable conditions (age, season, parity etc.), the genetic evaluation process had to distinguish the breeding value from all environmental effects that were nuisance factors in this context. Dairy cattle 
was the first species to be analyzed using Best Linear Unbiased Prediction in order to estimate breeding values in an accurate and unbiased way. Applied to an animal model, this statistical procedure also accounts for all relationships between animals, and for assortative mating.

This strategy based on progeny testing was very efficient, with a steady-state yearly genetic trend of about 0.2 genetic standard deviation of the breeding objective. An important drawback was the limited number of bulls used as sires of cows, and even more markedly as sires of bulls. This resulted in a low effective size of all dairy breeds, close to 50 , even for large populations with millions of cows. In most dairy breeds, the ten highest contributing ancestors to the current populations contribute around $50 \%$ of the genes to the current generation. As a consequence, inbreeding trends were around $1 \%$ per generation and regular emergence of genetic defects were observed in nearly all breeds. In many breeds, the genetic trend has been more important for heritable traits such as production or conformation, whereas some functional traits such as fertility and disease resistance have been reduced or barely maintained.

\section{The genomic revolution}

In 2001, Meuwissen et al. proposed a practical method to predict the breeding value of animals from their genotypes on a large number of genetic markers spanning their whole genome. The idea was to estimate the statistical effects of these markers (without any assumption on their biological effects), from a large reference population of animals with both phenotypic and genotypic information. Then these estimated effects were applied to candidates genotyped for the same markers. These candidates could be evaluated as soon as a molecular analysis can be carried out, that is very early in life. Initially, the limiting factor was the lack of genotyping tools at a reasonable cost. After some initial trials showing that the method worked, a suitable SNP chip was proposed in 2007 by Illumina with 54000 markers (Matukumalli et al., 2009), opening the way for practical genomic selection.

Indeed, the conditions to efficiently apply genomic selection are all met in dairy cattle. In 2006, Schaeffer showed that the genetic trend could be doubled at a lower cost than the conventional progeny-test scheme. This situation is much more favourable than in other species for which a 10-30\% increase in genetic gain is often predicted but at a higher cost. In dairy cattle, this doubled genetic gain is due to a strong decrease in generation interval obtained by giving up progeny testing, which was an especially long procedure. Indeed, future bulls can be selected in the first month of life and used in artificial insemination as soon as they can produce semen. This time-reduction aspect affects both the sire-son and sire-daughter selection pathways as well as the dam-son pathway if females are also genotyped. 
The much lower cost advocated by Schaeffer is controversial because it was subsequently shown that genomic selection generates new costs, (especially for reference population phenotyping) not fully anticipated initially. But in any case, genomic selection is not more expensive than conventional selection overall and it is much cheaper at the level of individual bulls. Indeed, the cost of a selected young bull ready for market is at least ten times lower than a service sire selected after progeny testing. This lower cost per bull provides a larger flexibility in the design of breeding schemes, especially regarding the number of bulls put on the market. This point will be developed later on.

Other characteristics are also very favou rable in dairy cattle. The genotyping cost is reasonable for such large animals, whereas it is much more critical for sheep, pigs or poultry. Most importantly, in the largest breeds, reference populations already existed via many progeny-tested bulls with frozen semen. The bull phenotypes were readily available, recorded as the average performance of their daughters (expressed as daughter-yield deviation, DYD). This DYD is equivalent to the bull's own performance, but with a high heritability, especially if the number of daughters is high enough, ensuring a high accuracy of genomic evaluation. This is true for all evaluated traits, even those with a low heritability. On the other hand, frozen semen was a source of DNA easy to access from a few centralized points. As a result, genomic selection could initially be implemented at a cost limited to obtaining genotypes, and resulted immediately in almost equivalent reliabilities of proofs for production traits, and higher reliabilities for some functional traits.

In practice, the shift from conventional to genomic selection has been very fast in the largest dairy breeds. The first genomic evaluations were released in 2009 in a number of countries such as the United States, Canada, France, Ireland, and New Zealand (VanRaden, 2008). All the benefits from genomic selection are obtained assuming an end to the progeny testing method, and that the only service sires used are young bulls with genomic evaluations, in order to fully take advantage of the reduction in generation interval. The decision of this remarkable switch, highly impacting the activity of breeding companies, varied a lot between countries. In France, for instance, progeny testing was stopped as early as 2009. In practice, it meant that no new young bull entered progeny testing after September 2009. Of course, all older bulls already enrolled in the process continued and obtained progeny-based estimated breeding values from 2010 to 2013. This provided a convenient opportunity for the industry to compare year after year the early genomic predictions with the conventional progeny-based breeding values. These four batches of results provided satisfactory validation, and increased the confidence of the industry and the farmers in the reliability of genomic evaluations. As the top ranking of bull lists were filled by young bulls, the semen market switched rapidly from progeny-tested bulls to young bulls. In France, the insemination market share of young bulls with genomic evaluations was already 20\% in 2010 and reached 75\% in 2014. 


\section{A major change in organization}

As a direct consequence of genomic selection, the number of bulls owned by the breeding companies decreased significantly, with a strong influence on their infrastructure. Many older bulls became obsolete and generated financial losses. Now, artificial insemination centres had to produce semen from more bulls simultaneously, and from younger pubescent bulls with specific constraints. Marketing also had to be adapted to these very different genetic products to make farmers confident in their quality.

It appeared very quickly to all breeders that a major parameter for the success of genomic selection was the size of the reference population. A number of breeding companies merged in order to create bigger entities. For conventional traits, new alliances emerged, aimed at merging several reference populations to build larger ones. In the case of Holstein cattle, two large consortiums were created. This initially involved merging reference populations from North America, the United States and Canada, (later also joined by the United Kingdom and Italy). This was immediately followed by a group of six, then eight, European countries to build Eurogenomics. These consortia benefited from the international evaluations carried out by Interbull that provided comparable phenotypes in a straightforward way. Lund et al. (2011) estimated that the gain in reliability reached 0.11 on average over all traits and countries within Eurogenomics, when this consortium was created in 2010 with a reference population of 16000 progeny-tested bulls (rising to 36000 in 2018). A similar strategy was used in the Brown Swiss breed to create the Intergenomics consortium, including seven countries and Interbull. More recently, a new consortium was created in Holstein through Interbull, gathering a group of smaller partners around Ireland following a collaboration framework initially developed in the Brown Swiss breed. Bilateral exchanges were also organized, for example between Danish and US Jersey breeds. Frequently these consortia were the framework of additional shared activities such as common research and development, standardization of information, imputation of missing genotypes, use of whole-genome sequence data, and phenotyping of new traits. Eurogenomics also acted as a unique channel for common purchase of large quantities of SNP chips, giving access to lower genotyping costs and later to customized chips.

But if information sharing was the major change for large international breeds and common traits, genomic selection also generated more private strategies, especially regarding the selection of new traits. Indeed, de novo assembling a reference population is a large investment to produce genomically enhanced breeding values (GEBV) for new traits. If these GEBV are a competitive advantage or a source of differentiation, breeders do not want to share this resource, or only with a closed circle of allied partners. Consequently, genomic selection has become both more international and more private (Labatut et al., 2014). 
Breeding companies tended also to merge or to develop alliances with performance recording organizations, with the objective of having better control over the phenotypes recorded, and to expand the number of recorded traits. Because a reference population created de novo is a significant investment, they developed specific and strong relationships with a selected network of partner farms to record new traits and to develop and test new technologies. Because bull dams are increasingly selected on their genomic breeding value at an early age, they now originate from a much larger number of farms than before.

\section{New breeding objectives and genetic trends}

As the expected genetic trend was doubled, it was clear for each breeder that the breeding objective should be changed. Indeed, it was anticipated that doubling the genetic trend for milk production, already very high, was not acceptable and would have led to considerable challenges in adapting the production system accordingly. Therefore, in many countries, the breeding objective was redirected with the idea to maintain the current genetic trend for production traits, and use the available selection intensity to efficiently select for functional traits and, potentially, for new traits. The effect on functional traits has been substantial, with good progress on fertility and mastitis resistance after decades of deterioration. For instance, in French Holstein, the economic weight of production was reduced from $50 \%$ to $35 \%$, whereas the overall weight of fertility and udder health (i.e. clinical mastitis and somatic cell counts) increased from $25 \%$ to $40 \%$.

Functional traits not only benefited from higher weights in the breeding objective but also from higher reliability of genomic estimated breeding values. In fact, with genomics, the reliability of proofs decreased slightly for production and increased for functional traits. This reduction in the range of reliability across traits was also favourable for a better balance in genetic trends across traits. This reduction in reliability range resulted directly from the fact that the reference population was made of progeny-tested bulls, with relatively high reliability of proofs for all traits. Moreover, with genomic evaluation, reliabilities are the same for both sexes. In other words, 1-year-old bull dams are now selected based on GEBV on fertility and other low heritability traits with the same reliability as old bulls with dozens of daughters!

Because the Al bull's cost is lower than before, more bulls can be proposed to satisfy a larger range of objectives. Therefore, we can anticipate a stronger segmentation of the semen market. For instance, pasture-based systems will demand less emphasis on stature and more on fertility than indoor systems. According to the environment, the breeding objective may be customized as well as the profile of the bulls used. 
In the future, not only will overall GEBV be estimated for a given trait, but also for one or several components varying with the environment, therefore including genetic $x$ environment interactions. These values may also contribute to market segmentation. Generally, any clear long-term objective representing a given market share of cattle genetics, may justify the production of dedicated bulls by breeding companies. Such a policy would also be favourable to genetic diversity, as discussed in the next section.

\section{Genomic selection and genetic diversity}

Conventional selection relied on a small number of elite bulls, resulting in a fast erosion of genetic variability. Because progeny testing was expensive, the probability of selection was maximized by choosing young bulls from the best sires and the best dams born from the best maternal grandsires. In contrast, it was initially argued that since genomic information allows the prediction of individual Mendelian sampling effect for candidates, dependence on family information is reduced when utilizing genomic selection. Indeed, at the expense of only additional genotypes, a large population of young males could be screened widely and the best bulls could be selected, independently from their origin. Therefore, many hopes were expressed for a better management of genetic diversity with genomic selection.

In practice, the situation is not as favourable and a more proactive policy is required to control inbreeding. As generation interval is reduced, if nothing else is changed, the yearly inbreeding trend is increased accordingly. But the situation is often much worse. If every year the breeding companies market the same number of new bulls as were in the conventional system, the total number of bulls per generation is reduced since the generation interval is shorter, strongly reducing the effective size of the population. Therefore, under this assumption, the yearly inbreeding trend varies with the inverse of the squared generation interval. In other words, if the generation interval is divided by 2 , the yearly inbreeding trend may be multiplied by 4 ! This means, it is essential to manage the populations under selection differently to control inbreeding trends.

As mentioned previously, the cost per bull is relatively low and this provides some flexibility. The reduced generation interval must be compensated for by a larger number of bulls, which is economically possible because bulls are cheaper. The short-term profitability of the breeding companies will be lower but the long-term profitability for the population will be higher. Colleau et al. (2015) showed that different scenarios without a progeny test may provide similar genetic gains, but very different inbreeding trends. The best scenario decreased inbreeding trends by $20 \%$ in comparison with the conventional scheme. In this scenario, a large number of bulls are used as soon as possible 
for a limited period of time, and with a limited total number of semen doses (10000 semen straws during 6 months). Each bull should also be used as the bull sire to generate one selected son in the next generation. Any other scenario with fewer bulls provides poor and often very alarming results in terms of inbreeding. For instance, when the semen market consists of $50 \%$ of semen from young bulls and $50 \%$ from the best 'old' bulls evaluated via progeny testing, the inbreeding trend is doubled in comparison with the conventional scheme.

Applying this recommended policy with many young bulls used during a short period is another cultural revolution in a world where elite bulls are very popular. Indeed, many young bulls with a short and limited use tend to become anonymous. The bull list needs to be updated two or three times a year. Farmers and technicians can no longer remember pedigrees, and computer tools are necessary to manage information and plan matings. New commercial policies have to be invented, based on 'packs' of bulls or profiles: the farmer does not buy a particular bull but an average genetic level for the breeding objective he himself defines.

We have to admit that these recommendations have been rarely followed. Due to cultural and commercial pressures, the number of bulls put on the market has been constantly reduced and less than 10 years after genomic selection was implemented, inbreeding trend shows a marked increase in the Holstein breed in different countries. In France, the situation is more satisfactory in Montbéliarde and Normande breeds, which are less constrained by international competition and, until now, have followed the proposed rules.

In smaller breeds such as the French Abondance (30000 cows), the situation has been very critical for a long time. In the previous conventional scheme, 10-15 bulls were progeny tested each year and 2-3 of them were selected as service bulls. These low numbers already created a serious loss in genetic effective size. Genomic selection (assuming a reference population can be assembled, see next section) represents a convenient solution. Indeed, it is technically and economically possible to market 8-10 new young bulls each year and therefore regenerate genetic diversity.

\section{Female genotyping}

Genomic evaluations have the same cost, and result in a similar accuracy for males and females. Initial genotyping efforts were focused mainly on males, either to assemble highly informative reference populations or to select bull candidates. A strong limitation for expanding genotyping activity was the cost of the chip.

However, female genotyping has a strategic importance at both farm and population level. At population level, it is the only way to replace the initial 
male reference population, or to assemble a de novo reference population for a smaller breed or a new trait. It is also the only way to generate large amounts of genotyping and therefore to dramatically decrease the price of genotyping. At farm level, jointly with the use of sexed semen, it provides new tools to efficiently select within the herd.

Therefore, in the beginning of the 2010s, a number of organizations worked to meet the conditions for large-scale genotyping, especially by reducing its cost. In 2012, a low-density chip was proposed with 6909 SNPs (Boichard et al., 2012). This chip was significantly cheaper than the 50k chip, and the missing information could be recovered by imputation with a high accuracy. Very large efforts were dedicated internationally to develop efficient imputation algorithms, more applicable to the large cattle populations with a strong family structure than the already existing algorithms developed in human genetics (e.g. Sargolzaei et al., 2014). This LD chip, well suited to all taurine breeds, was used on a very large scale, and has been the major engine for cost reduction, allowing female genotyping expansion. However, a virtuous circle was created, a lower price induced a larger market and higher amounts decreased the cost. The most impressive example is seen in Ireland, with a very large genotyping programme targeting $100 \%$ of the multi-breed population at a very low cost. Therefore, with the increase in volume, genotyping cost is less and less of an issue and will probably continue to decrease. Consequently, it is foreseen that in the very near future, genotyping will be common practice for the majority of dairy heifers.

\subsection{For construction of a reference population}

In large breeds, male reference populations were initially assembled by genotyping one or two generations of progeny-tested bulls. Genomic selection relied on past and not renewable data. Because extensive progeny testing of many bulls is expected to stop, the number of new bulls with progeny will decrease and male reference populations will be difficult to replace. Therefore, the reference populations will evolve and gradually be replaced by female or mixed populations. However, females are less informative than progeny-tested males. Corresponding reference populations must be larger to achieve the same prediction accuracy (Boichard et al., 2015). On average, 3-10 times more cows are required according to the heritability of the traits, and this figure can even be much higher for low heritability traits. Therefore, to ensure the sustainability of genomic selection beyond the initial effort, it is essential to build the favourable conditions for large-scale female genotyping and phenotyping.

This can be a particularly challenging situation for breeds with smaller population sizes. Indeed, male reference populations are often suboptimal 
in medium-size breeds (e.g. around 2500 progeny-tested bulls in the Montbéliarde reference population over 15 years in France) and critically low in many regional breeds (e.g. 250 bulls available in the French Abondance breed). These situations cannot be improved through additional informative males because all Al males have already been genotyped and included in the reference population. Many of them do not have large closely-related populations in other countries to build an international consortium. Until now, attempts to combine reference populations from different breeds have provided little gain in GEBV accuracy for practical selection purposes (Erbe et al., 2012; Hozé et al., 2014), and it is difficult to anticipate the efficiency of future multi-breed approaches using whole-sequence information (Daetwyler et al., 2014). Therefore, in the short term, the most reliable alternative is to extend the reference populations to females. Such a strategy has been applied in different medium-size populations, for instance in Jersey and Red breeds in Denmark (Thomasen et al., 2014). In France, it has been implemented in the Montbéliarde and Normande breeds, as well as in smaller populations such as the Abondance, Tarentaise and Vosgienne breeds (Jonas et al., 2017). In the Montbéliarde breed, female genotyping is very much developed: more than 50000 cows with phenotypes were included in the reference population in 2017 and this figure continues to grow rapidly. In smaller breeds, genomic selection is relatively much more expensive. The fixed cost of the reference population is high and is not fully compensated for by the decrease in expenses for bulls, which are more limited than in large breeds. In addition, where genomic selection is not yet applied in small breeds, there is no interest for the farmers to genotype their cows. Therefore the initial high investment for genotyping a reference population is possible only through cost sharing between farmers, breed associations and breeding companies, and with the help of local and national funding bodies. Genomic selection was implemented in Abondance, Tarentaise and Vosgienne breeds in 2016. Its accuracy is of course significantly lower than in the larger breeds, but is high enough to select bulls. In these breeds, the number of doses is limited to a few thousands per Al bull.

In many emerging or developing countries, the general infrastructure for a large-scale identification and pedigree and performance recording does not exist. In some cases, huge investments have been made during a limited period with international support but proved to be unsustainable. Genomic selection based exclusively on a female reference population is a very attractive, and a more realistic direction to finally undertake a sustainable selection of local breeds in those countries, for example in India (Ducrocq et al., 2018). It would rely on collecting performances and biological samples from a few thousand cows, even from very small herds. Of course, this requires adequate funding, adapted logistics, proper attention to adaptation traits and genotype $\times$ environment interactions, as well as appropriate scientific support. The resulting reliability of $\mathrm{Al}$ 
or natural service bulls is likely to be quite low but would still be an improvement - and this makes a huge difference with all the unsuccessful past experiments.

\subsection{For within-herd selection}

The genotyping information of all cows within a herd gives access to new and profitable applications. These include completely accurate information about parentage, and a greatly improved mating plan accounting for accurate GEBV for both males and females on many traits and genetic defects (Cole, 2015). Genomic inbreeding coefficients can be provided for all females. Indicators of 'genetic originality' can be computed from genotypes, relative to the rest of the herd and to the breed, to monitor genetic diversity at herd or breed level. New monitoring systems integrating genotyping are being developed. A useful example is the estimation of individual somatic cell counts by genotyping a milk sample from the bulk tank (Blard et al., 2012).

But the major criterion for genotyping profitability is its potential for within-herd selection. Within-herd selection on the dam-daughter pathway has always been inefficient, due to the conjunction of both low EBV accuracy and low reproductive capacity. Female genotyping provides a high GEBV accuracy, whereas the use of sexed semen allows a choice of replacement heifers from the better half of the herd. Therefore, coupling female genotyping with the use of sexed semen provides an efficient solution for within-herd selection. The use of sexed semen is usually profitable by itself, due to the higher value of female calves or crossbred offspring than of purebred male calves. Genotyping profitability depends on genotyping cost. It was demonstrated that a positive return due to extra genetic gain is expected after the first or second lactation of the progeny, if genotyping costs are less than 20-40€ according to economic conditions (Boichard et al., 2013; Hjorto et al., 2015; Newton et al., 2018). In most countries, genotyping costs were initially higher than the break-even cost. In addition, a delay of several years after the initial investment is required before a positive return is obtained, and this limits the development of genotyping. But the situation is rapidly evolving with a decrease in genotyping cost and the development of technical applications adapted to this new information. Because of the common interest of genotyping for farmers, breed associations and breeding companies, genotyping costs can also be shared between the different parties.

For the first time, female genotyping also opens new avenues for breeding objective customization. Dams can be accurately selected on total merit indices defined by the farmer, adapted to his own conditions, with more emphasis on, for example, either production, type, functional traits or milk composition. This within-herd selection can be combined with the choice of Al bulls, from a much larger panel and more diverse profiles than before. Additionally, as mentioned 
before, mating decisions can integrate accurate GEBV, genetic defects and relationship information. GEBV will be adapted to local conditions by accounting for genetic $\times$ environment interactions. Finally, large-scale genotyping is also a good way to screen the population for bull dams with a direct benefit for the farmer as well as strong efficiency - no preferential treatment possible - and limited risk of bias for the breeding scheme. It seems likely that an increasing proportion of dairy heifers will be genotyped in the near future.

\section{Selection for new traits}

Genomics provides unprecedented potential selection for new traits. Benefits include, (1) as previously indicated, potential genetic gain is doubled, leaving room for new traits without giving up attention to the main traits; (2) large-scale performance recording is no longer required and a reference population of, for example, several thousand records per year makes selection feasible on the new traits; (3) there are clear motivations to account for new traits in selection: the environmental impact of animal production, the need to decrease the use of drugs and antibiotics, the multiple demands of the consumers, and the need to adapt to global change. Breeding companies also may want to differentiate themselves from their competitors and to propose specific genomic evaluations not yet available with others.

There are many interesting possibilities (Boichard and Brochard, 2012), the most attractive ones rely on the use of already existing data. Connecting existing phenotypes to cow genotypes generates reference populations at a marginal cost. Several examples can be given.

- Milk is routinely analysed with mid-infrared spectrometers for basic composition (fat, protein). But the potential of milk spectra is much higher and many efforts have been devoted to predicting detailed milk composition (fatty acids profile, individual proteins, lactose, minerals and citrate), milk properties (cheese yield, coagulation properties) and other traits relative to the cows (ketosis, energy balance and methane emission). Although these phenotypic predictions are not all accurate, the repeated measures per cow and the large number of records in the population help to derive suitable genomic predictions.

- Carcass traits(including weight, conformation and fatness) may be available from slaughterhouse databases. Because many males are genotyped by breeding companies, genomic evaluations can be obtained for carcass traits of young calves and steers. But the best potential is for culled cows if a large number of them are genotyped.

- In many countries, disease events are recorded by the farmers. This information is recorded for herd management and sanitary traceability. 
After data centralization, even if many of them need to be filtered out for lack of quality, huge amounts of data should remain available for genomic prediction. Targeted diseases are common ones such as clinical mastitis, metritis, retained placenta and metabolic disorders.

- Merging performance data in geo-localized farms with already existing climatic information makes it possible to estimate genetic $\times$ environment interactions. A direct application can be the evaluation of heat tolerance, through a reaction norm genomic model of performances described as a function of temperature and humidity (Hayes et al., 2009).

In other situations, data can be recorded during already existing nongenetic activities. For instance, in herds affected by paratuberculosis, ELISA and PCR tests carried out for disease diagnosis and control can be used as phenotypes for genomic predictions. Again, some rigorous data filtering is required to maximize the relationship between the phenotype and the true status, infected or uninfected after exposition. Another example is foot and leg lesions which are scored in a standardized way during hoof trimming and these records can also be used for genomic evaluation (Croué et al., 2017).

Finally, some very important traits do not have any known proxy which is easy to record, and therefore need a dedicated phenotyping effort. This is the case for feed efficiency which requires extensive measures of feed intake over several months. Such a recording can now be automated, thereby reducing the labour load. However, the investment required per cow is very high, justifying international collaborations and data sharing to assemble a large reference population and obtain a high prediction accuracy (Berry et al., 2014).

\section{Conclusion and future trends}

The dairy genetic industry has faced a revolution with dramatic changes of its tools and practices. This trend is not yet completed and other significant advances are expected in the future. Some evolutions may simplify the overall design, such as the multi-breed evaluations. Many efforts are devoted worldwide to integrate whole-genome sequence data, causal variants (or variants very close to them), and functional annotations into prediction models. If these studies are successful, we may anticipate that reference populations will be shared across breeds, limiting the fixed cost of the investment for their creation or replacement. Similarly, such future evaluations are expected to produce more persistent predictions, reducing the constraint of reference population maintenance. With the noticeable exception of New Zealand, the development of crossbreeding is still limited in dairy cattle. However, crossbreeding may present many advantages, especially for a more sustainable production. Dairy cattle could benefit from the experience gained with other species (such as in pig) to expand genomic predictions in a crossbreeding context. 
Breeding objectives will continue to evolve and to include increasingly diverse traits. After a transition period marked by some excess in inbreeding trends, we hope for a better management of genetic diversity with more bulls used as sires of daughters and sires of bulls. Genomic information will be available for an increasing proportion of the population, including females. This will generate new practices, especially within herd (optimized mating plans, selection accounting for $\mathrm{G} \times \mathrm{E}$ interactions, and optimization of replacement and culling). The impacts of other new technologies are difficult to fully anticipate. Applications based on epigenetic control of the variability or microbiota orientation have not yet been designed, while gene editing remains a controversial topic. Several studies showed that gene editing combined with genomic selection can significantly enhance genetic trends (Jenko et al., 2015) at the expense of a much higher inbreeding trend, but consumer acceptance is still an open question.

The general organization of dairy cattle breeding has been strongly affected by genomic selection and this trend will likely continue. Whereas progeny testing required large populations and many herds both as contributors and users of genetics, genomic selection requires only good reference populations with phenotypes and genotypes, and an easy access to the best bulls. The reference population, of limited size, can be linked to breeding companies on a contractual basis. Therefore, breeding companies will be increasingly organized as private companies, even if many of them will remain cooperatives. In parallel, we see the emergence of large private companies proposing genomic solutions. For the largest populations, it is anticipated that a few select companies will dominate the world market of genetics. This new structure will clearly be a threat to the traditional country-based organizations, as well as to international organizations such as Interbull.

\section{Acknowledgements}

Didier Boichard thanks Vincent Ducrocq, Sebastien Fritz, Sophie Mattalia, JeanJacques Colleau, Pascal Croiseau and Julie Labatut for extensive discussions and useful comments on the manuscript.

\section{References}

Berry, D. P., Coffey, M. P., Pryce, J. E., de Haas, Y., Lovendahl, P., Krattenmacher, N., Crowley, J. J., Wang, Z., Spurlock, D., Weigel, K., et al. 2014. International genetic evaluations for feed intake in dairy cattle through the collation of data from multiple sources. J. Dairy Sci. 97(6), 3894-905. doi:10.3168/jds.2013-7548.

Blard, G., Zhang, Z., Coppieters, W. and Georges, M. 2012. Identifying cows with subclinical mastitis by bulk single nucleotide polymorphism genotyping of tank milk. J. Dairy Sci. 95(7), 4109-13. doi:10.3168/jds.2011-5178.

Boichard, D. and Brochard, M. 2012. New phenotypes for new breeding goals in dairy cattle. Animal 6(4), 544-50. doi:10.1017/S1751731112000018. 
Boichard, D., Chung, H., Dassonneville, R., David, X., Eggen, A., Fritz, S., Gietzen, K. J., Hayes, B. J., Lawley, C. T., Sonstegard, T. S., et al. 2012. Design of a bovine lowdensity SNP array optimized for imputation. PLoS ONE 7(3), e34130. doi:10.1371/ journal.pone.0034130.

Boichard, D., Dassonneville, R., Mattalia, S., Ducrocq, V. and Fritz, S. 2013. All cows are worth to be genotyped! Interbull Meeting, 24-30 August 2013, Nantes. Interbull Bulletin No. 47, pp. 256-60.

Boichard, D., Ducrocq, V. and Fritz, S. 2015. Sustainable dairy cattle selection in the genomic era. J. Anim. Breed. Genet. 132(2), 135-43. doi:10.1111/jbg.12150.

Cole, J. B. 2015. A simple strategy for managing many recessive disorders in a dairy cattle breeding program. Genet. Sel. Evol. 47(1), 94. doi:10.1186/s12711-015-0174-9.

Colleau, J. J., Fritz, S., Guillaume, F., Baur, A., Dupassieux, D., Boscher, M. Y., Journaux, L., Eggen, A. and Boichard, D. 2015. Simulating the potential of genomic selection in dairy cattle. INRA Prod. Anim. 28, 251-8 (in French).

Croué, I., Fikse, F., Johansson, K., Carlén, E., Thomas, G., Leclerc, H. and Ducrocq, V. 2017. Genetic evaluation of claw health traits accounting for potential preselection of cows to be trimmed. J. Dairy Sci. 100(10), 8197-204. doi:10.3168/jds.2017-13002.

Daetwyler, H. D., Capitan, A., Pausch, H., Stothard, P., van Binsbergen, R., Brøndum, R. F., Liao, X., Djari, A., Rodriguez, S. C., Grohs, C., et al. 2014. Whole-genome sequencing of 234 bulls facilitates mapping of monogenic and complex traits in cattle. Nat. Genet. 46(8), 858-65. doi:10.1038/ng.3034.

Ducrocq, V., Laloe, D., Swaminathan, M., Rognon, X., Tixier-Boichard, M. and Zerjal, T. 2018. Genomics for ruminants in developing countries: from principles to practice. Front. Genet. 9, 251. doi:10.3389/fgene.2018.00251.

Erbe, M., Hayes, B. J., Matukumalli, L. K., Goswami, S., Bowman, P. J., Reich, C. M., Mason, B. A. and Goddard, M. E. 2012. Improving accuracy of genomic predictions within and between dairy cattle breeds with imputed high-density single nucleotide polymorphism panels. J. Dairy Sci. 95(7), 4114-29. doi:10.3168/jds.2011-5019.

Hayes, B. J., Bowman, P. J., Chamberlain, A. J., Savin, K., van Tassell, C. P., Sonstegard, T. S. and Goddard, M. E. 2009. A validated genome wide association study to breed cattle adapted to an environment altered by climate change. PLoS ONE 4(8), e6676. doi:10.1371/journal.pone.0006676.

Hjorto, L., Ettema, J. F., Kargo, M. and Sorensen, A. C. 2015. Genomic testing interacts with reproductive surplus in reducing genetic lag and increasing economic net return. J. Dairy Sci. 98(1), 646-58. doi:10.3168/jds.2014-8401.

Hozé, C., Fritz, S., Phocas, F., Boichard, D., Ducrocq, V. and Croiseau, P. 2014. Efficiency of multi-breed genomic selection for dairy cattle breeds with different sizes of reference population. J. Dairy Sci. 97(6), 3918-29. doi:10.3168/jds.2013-7761.

Jenko, J., Gorjanc, G., Cleveland, M. A., Varshney, R. K., Whitelaw, C. B. A., Woolliams, J. A. and Hickey, J. M. 2015. Potential of promotion of alleles by genome editing to improve quantitative traits in livestock breeding programs. Genet. Sel. Evol. 47(1), 55. doi:10.1186/s12711-015-0135-3.

Jonas, D., Ducrocq, V., Fritz, S., Baur, A., Sanchez, M.-P. and Croiseau, P. 2017. Genomic evaluation of regional dairy cattle breeds in single-breed and multi-breed contexts. J. Anim. Breed. Genet. 134(1), 3-13. doi:10.1111/jbg.12249.

Labatut, J., Astruc, J. M., Barillet, F., Boichard, D., Ducrocq, V., Griffon, L. and Lagriffoul, G. 2014. Organizational implications of genomic selection in French dairy cattle and sheep: from diagnosis to a collective action. INRA Prod. Anim. 27, 303-16 (in French). 
Lund, M. S., Roos, A. P., Vries, A. G., Druet, T., Ducrocq, V., Fritz, S., Guillaume, F., Guldbrandtsen, B., Liu, Z., Reents, R., et al. 2011. A common reference population from four European Holstein populations increases reliability of genomic predictions. Genet. Sel. Evol. 43, 43. doi:10.1186/1297-9686-43-43.

Matukumalli, L. K., Lawley, C. T., Schnabel, R. D., Taylor, J. F., Allan, M. F., Heaton, M. P., O'Connell, J., Moore, S. S., Smith, T. P. L., Sonstegard, T. S., et al. 2009. Development and characterization of a high density SNP genotyping assay for cattle. PLoS ONE 4(4), e5350. doi:10.1371/journal.pone.0005350.

Meuwissen, T. H. E., Hayes, B. J. and Goddard, M. E. 2001. Prediction of total genetic value using genome-wide dense marker maps. Genetics 157, 1819-29.

Newton, J. E., Hayes, B. J. and Pryce, J. E. 2018. The cost-benefit of genomic testing of heifers and using sexed semen in pasture-based dairy herds. J. Dairy Sci. 101(7), 6159-73. doi:10.3168/jds.2017-13476.

Sargolzaei, M., Chesnais, J. P. and Schenkel, F. S. 2014. A new approach for efficient genotype imputation using information from relatives. BMC Genomics 15(1), 478. doi:10.1186/1471-2164-15-478.

Schaeffer, L. R. 2006. Strategy for applying genome-wide selection in dairy cattle. J. Anim. Breed. Genet. 123(4), 218-23. doi:10.1111/j.1439-0388.2006.00595.x.

Thomasen, J. R., Sorensen, A. C., Lund, M. S. and Guldbrandtsen, B. 2014. Adding cows to the reference population makes a small dairy population competitive. J. Dairy Sci. 97(9), 5822-32. doi:10.3168/jds.2014-7906.

VanRaden, P. M. 2008. Efficient methods to compute genomic predictions. J. Dairy Sci. 91(11), 4414-23. doi:10.3168/jds.2007-0980. 